\title{
A Two-Arm Situated Artificial Communicator for Human-Robot Cooperative Assembly
}

\author{
Jianwei Zhang, Member, IEEE, and Alois Knoll
}

\begin{abstract}
We present the development of a robot system with some cognitive capabilities, as well as experimental results. We focus on two topics: assembly by two hands and understanding human instructions in nonconstrained natural language. These two features distinguish human beings from animals, and are, thus, the means leading to high-level intelligence. A typical application of such a system is a human-robot cooperative assembly. A human communicator sharing a view of the assembly scenario with the robot instructs the latter by speaking to it in the same way that he would communicate with a child whose common-sense knowledge is limited. His instructions can be underspecified, incomplete, and/or context dependent.

After introducing the general purpose of our research project, we present the hardware and software components of our robots needed for interactive assembly tasks. We then discuss the control architecture of the robot system with two stationary robot arms by describing the functionalities of perception, instruction understanding, and execution. To show how our robot learns from humans, the implementations of a layered learning methodology, memory, and monitoring functions are introduced. Finally, we outline a list of future research topics related to the enhancement of such systems.
\end{abstract}

Index Terms-Cognitive science, cooperative systems, learning control systems, multiple manipulators, natural language interfaces.

\section{INTRODUCTION}

$\mathbf{E}$ NDOWING a robot system with the ability to carry on a goal-directed multimodal dialogue using natural language $(\mathrm{NL})$, speech, gesture, gaze, etc., for performing nontrivial tasks is a demanding challenge not only from a robotics and a computer science perspective, but it cannot be tackled without a deep understanding of linguistics and human psychology [5]. There are two conceptually different approaches to designing an interface architecture for incorporating NL input into a robotic system: the Front-End and the Communicator approaches.

\section{A. Front-End Approach}

The robot system receives instructions in NL that completely specify a task the instructor wants to be performed. The input is analyzed and the necessary actions are taken in a subsequent

Manuscript received January 7, 2002; revised September 9, 2002. Abstract published on the Internet May 26, 2003. This work was supported by the Deutsche Forschungsgemeinschaft (DFG), the German Research Council, under Grant SFB 360. This paper was presented at the 2001 IEEE International Workshop on Robot and Human Interactive Communication, Bordeaux and Paris, France, September 18-21.

J. Zhang is with the Department of Computer Science, University of Hamburg, 22527 Hamburg, Germany (zhang@informatik.uni-hamburg.de).

A. Knoll is with the Department of Computer Science, Technical University of Munich, 81667 Munich, Germany (knoll@informatik.tu-muenchen.de).

Digital Object Identifier 10.1109/TIE.2003.814767 separate step. Upon completion of the task, i.e., after having carried out a script invoked by the instruction fully autonomously, the system is ready for accepting new input. This approach is ideal for systems that have to deal only with a limited set and scope of tasks, which do not vary much over time either. Inadvertent changes of the environment resulting from the robot's actions, which would require a reformulation of the problem, cannot be considered. Neither is it possible to make specific references to objects (and/or their attributes) that are relevant only to certain transient system states because neither the programmer nor the instructor can foresee all of these states. Examples of this approach can be found in [1], [6], and [10].

To overcome the limitations of this approach, the concept of the "Artificial Communicator" was developed, which we briefly outline in the following.

\section{B. Communicator Approach}

If the nature of assembly tasks cannot be fully predicted, it becomes inevitable to decompose them into more elementary actions. Ideally, the actions specified are elementary in such a way that they always refer to only one step in the assembly of objects or aggregates, i.e., they refer to only one object that is to be assembled with another object or collection of aggregates. The entirety of a system that transforms suitable instructions into such actions is called an Artificial Communicator (AC). It consists of sensor subsystems, NL processing and further cognitive modules, and the robotic actors. From the instructor's point of view the AC should resemble a Human Communicator $(H C)$ as closely as possible [8]. This implies several important properties of AC behavior.

1) All modules of the $\mathrm{AC}$ must contribute to an event-driven incremental behavior: as soon as sufficient NL input information becomes available, the $\mathrm{AC}$ must react. Response times must comply with human waiting tolerances.

2) One of the most difficult problems is the disambiguation of instructor's references to objects. This may require the use of sensor measurements such as integration of robot vision or further $\mathrm{NL}$ input resulting from an $\mathrm{AC}$ request for more detailed information.

3) In order to make the system's response seem "natural," some rules of speech act theory should be observed. The sequence of actions must follow a "principle of least astonishment," i.e., in a given state the $\mathrm{AC}$ should take the actions that the instructor would expect it to take. Furthermore, sensor measurements and their abstractions that are to be communicated about must be transformed into a form comprehensible for humans. 
4) It must be possible for the instructor to communicate with the AC about both scene or object properties (e.g., object position, orientation, type) and about the AC system itself. Examples of the latter are meta-conversations about the configuration of the robot arms or about actions taken by the AC.

5) The instructor must have a view of the same objects in the scene as the AC's perception system.

6) The AC must exhibit robust behavior, i.e., all system states, even those triggered by contradictory or incomplete sensor readings as well as nonsensical NL input must lead to sensible actions being taken.

Altogether, the AC must be seamlessly integrated into the handling/manipulation process. More importantly, it must be situated, which means that the situational context (i.e. the state of the AC and its environment) of a certain NL and input of further modalities is always considered for its interpretation. The process of interpretation, in turn, may depend on the history of utterances up to a certain point in the conversation. It may be helpful, for example, to clearly state the goal of the assembly before proceeding with a description of the elementary actions. There are, however, situations in which such a "stepwise refinement" is counterproductive, e.g., if the final goal cannot be easily described. Studies based on observations of children performing assembly tasks have proven to be useful in developing possible interpretation control flows.

From the engineering perspective, the two approaches can be likened to open-loop control (Front-End Approach) and closed-loop control (Communicator Approach) with the human instructor being part of the closed loop. Several projects on communicative agents realized with real robots have been reported, e.g., [2] abd [9].

Our research work described in the following sections is embedded in a larger interdisciplinary research project aiming at the development of ACs for various purposes and involving scientists from the fields of computer linguistics, cognitive linguistics, computer science, and robotics. For performing assembly tasks and to facilitate human interaction with language and gestures, we have been developing a two-arm robotic system to model and realize human sensorimotor skills. This robotic system serves as the major test bed of the ongoing interdisciplinary research program of the project SFB ${ }^{1} 360$ "Situated Artificial Communicators" (SACs) at the University of Bielefeld, Germany [13].

\section{SAC}

There is ample evidence that there exists a strong link between human motor skill and cognitive development, e.g., see [7]. Our abilities of emulation, mental modeling, and planning of motion are central to human intelligence [3] and, by the way, a precondition for anticipation, but they also critically depend on the experience we make with our own body dynamics as we plastically adapt our body's shape to the environment. As a basic scenario, the assembly procedure of a toy aircraft (constructed with "Baufix" parts, see Fig. 1) was selected. A number

${ }^{1}$ Collaborative research centre funded by the Deutsche Forschungsgemeinschaft (DFG).

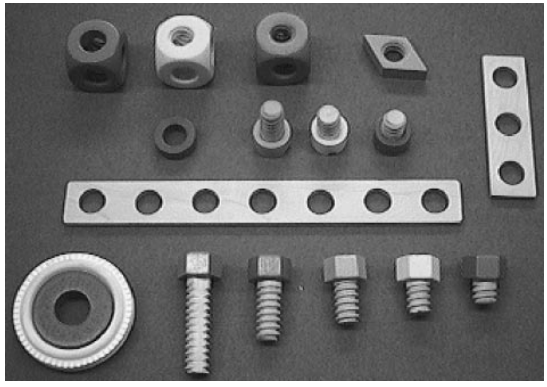

(a)

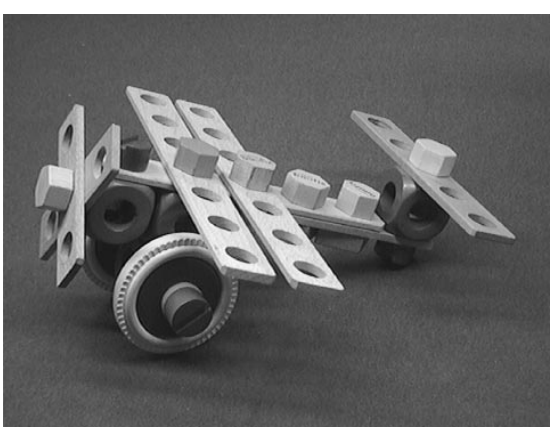

(b)

Fig. 1. Assembly of a toy aircraft. (a) Baufix construction parts. (b) Goal aggregate.

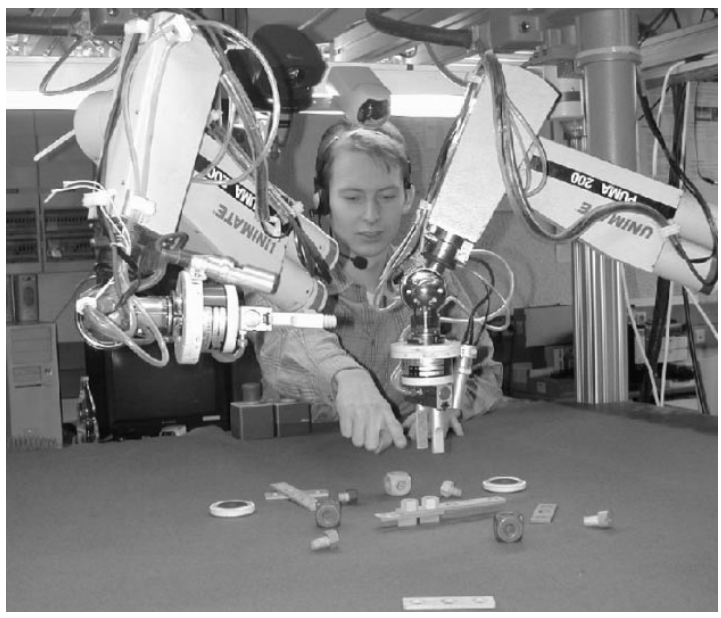

Fig. 2. Two-arm multisensor robot system for dialogue-guided assembly.

of assembly parts must be recognized, manipulated, and assembled to construct the model aircraft. In each of these steps, a human communicator instructs the robot, which implies that the interaction between them plays an important role in the whole process (Fig. 2).

The physical setup of the SAC system consists of the following components.

1) Two six-degrees-of-freedom PUMA-260 manipulators are installed overhead in a stationary assembly cell. On each wrist of the manipulator, a pneumatic jaw gripper with integrated force/torque sensor and "self-viewing" hand-eye system (local sensor) is mounted. As an option, a third manipulator with hand camera installed on the side can be applied to help with fixating or active exploration tasks. 


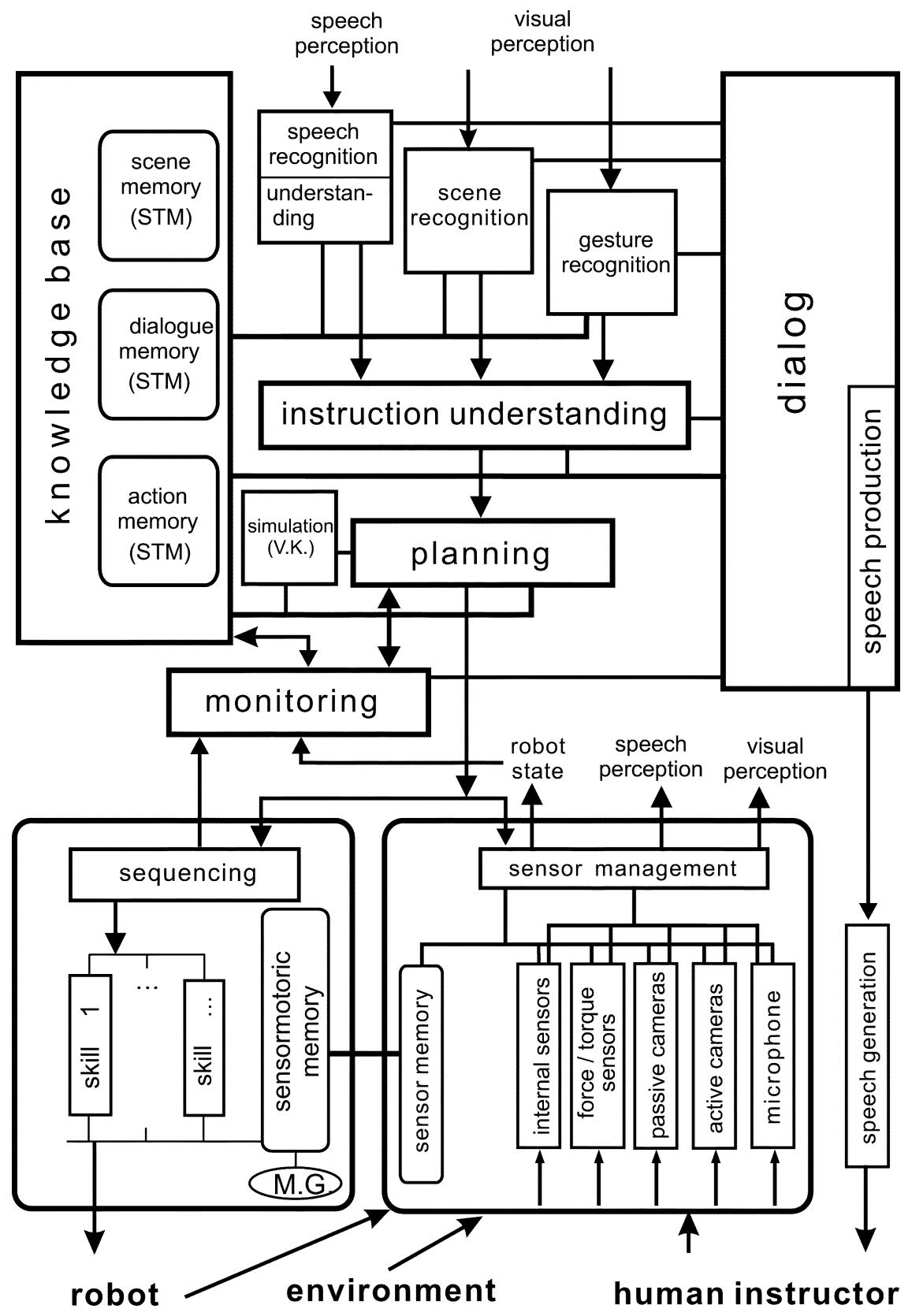

Fig. 3. Control architecture of the SAC for perception, instruction understanding, and execution.

2) Two cameras with controllable zoom, auto-focus, and auto-exposure provide the main vision function. Their tasks are to build two-dimensional (2-D)/three-dimensional (3-D) world models, to supervise gross motion of the robot as well as to trace the gesture and gaze of the human instructor.

3) A microphone and loudspeakers are connected with a standard voice recognition system to transform spoken instructions to word sequences and to synthesize the generated speech output.

\section{CONTROL ARChitecture}

As the backbone of an intelligent system, the control architecture of a complex technical system describes the functionality of individual modules and the interplay between them. We developed an interactive, incremental architecture for the SAC according to Fig. 3. An HC is closely involved in the whole assembly process.

For clarity, the whole architecture is partitioned into three blocks: Perception (right bottom), High-Level Cognitive Functions (upper half). and Execution (left bottom).

\section{A. Perception Modules}

The tasks of the perception system include self-perception and the perception of the physical environment as well as the human instructor. The complete robot state is specified by the joint and Cartesian positions of the arm, the posture of the hand, and the forces/torques exerted on the latter. This information can be acquired by the robot internal sensors like encoders/potentiometers and force/torque sensors. The current robot state is 
the input to the "monitoring" module. Another interesting topic on the supporting role of the robot state to help better understanding emotional intervention instructions like "Halt!" when the robot is moving near to the assembly surface. The assembly objects in the environment are observed by multiple cameras-a major function of sensor-based robotics.

To better handle the "human-in-the-loop" problem, human perception is viewed as one important extension of autonomous robots. Therefore, we track visual information about the human instructor like gesture and gaze with the static and articulated cameras. The naturally spoken instructions of the human instructor are input through a microphone and recognized as word sequences. The sensor management contains the data fusion and sensor integration, supplies the specified values of "robot state," "speech perception," and "visual perception." The speech and visual perception results are the main inputs for the high-level cognitive functions outlined below.

\section{B. High-Level Cognitive Functions}

The SAC and the HC interact through natural speech and a small set of hand gestures. First, an instruction is spoken to the robot system and recognized with the speech recognition engine. In the current system, ViaVoice recognizes only sentences which the grammar we developed allows. In practice, hundreds of grammatical rules can be used. If the recognition succeeds, the results are forwarded to the speech recognition/understanding module.

1) Transforming Instructions to Elementary Operations: By their very nature, human instructions are situated, ambiguous, and frequently incomplete. In most cases, however, the semantic analysis of such utterances will result in sensible operations. An example is the command "Grasp the left screw." The system has to identify the operation ( grasp), the object for this operation (screw), and the situated specification of the object (left). With the help of a hand gesture the operator can further disambiguate the object. The system may then use the geometric knowledge of the world to identify the right object. Other situated examples are: "Insert in the hole above," "Screw the bar on the downside in the same way as on the upside," "Put that there," "Rotate slightly further to the right," "Do it again," etc.

The output of the analysis is then verified to check if the intended operation can be carried out. If in doubt, the SAC asks for further specifications or it is authorized to pick an object by itself. Once the proper operation is determined, it is given to the execution module on the next level. The final result on this level consists of an Elementary Operation (EO) and the objects to be manipulated with the manipulation-relevant information such as type, position/orientation, color, and pose (standing, lying, etc).

An EO is defined in this system as an operation which does not need any further action planning. Typical EOs are: grasp, place, insert into, put on, screw, regrasp, and alignment. The robustness of these operations mainly depends on the quality of the different skills.

2) Planning and Monitoring: Based on the planning module, an assembly task of the toy aircraft, or of subaggregates, is decomposed into a sequence of EOs. The final decision about the motion sequence depends on the instructions of the human user as well as the generated plan. The planning module should not only be able to understand the human instructions, but it should also learn from the human guidance and improve its planning abilities gradually. It receives an EO from instruction understanding. By referencing the action memory, the planning module chooses the corresponding basic primitive sequence for the operation. This sequence is a script of basic primitives for implementing the given EO. The task here includes planning of the necessary trajectories, choosing the right $\operatorname{robot}(\mathrm{s})$ and basic exception handling.

Monitoring plays an important role in making an intelligent system robust. It is also used frequently by a human being in manipulation and speaking, especially in a new environment or for a new task. Monitoring and potential replanning for repair actions result in the nonlinearity of the understanding-planning-execution cycle, but they represent one essential function in the cognitive architecture of a robot. Furthermore, it is meaningful to add a diagnosis function which can provide hypotheses about the reasons of diverse failures.

The unexpected events during the robot action can be, for example: a force exceeds a defined threshold; a camera detects no object; singularities; collisions, etc. If such an event occurs, it is reported to the planning module.

The planning module receives an event report that is generated by the execution module described below. In normal operations, the monitoring module updates the action memory. It also detects the event failures. If it is found that the robot can continue and/or take repair actions, the planning module will generate an appropriate plan. Otherwise, the monitoring module sends a request to the dialogue module to ask the human communicator how to handle the exception and waits for an instruction. After the execution of each operation, the knowledge base is updated.

3) Memories: In the knowledge base, only semantic and procedural knowledge is used. In our current implementation this knowledge is still hard coded. It can be viewed as long-term memory to a certain degree, which will be extended by learning approaches in our future research. Short-term memories exist in perception modules, which are used for scene recognition, dialogue preparation, and action (sensorimotor functions). Learning of another important type of memories, the episodic memory, has been preliminarily studied for the assembly scenarios.

According to empirical investigations, episodic memory represents one of the most important components of human intelligence. Remember that mental simulation and planning use episodic memory as the basis. The diverse multisensor data with high bandwidth of our robot such as vision system, joint angles, positions, force profiles, etc., can obviously not be saved in their raw format for an arbitrarily long period of time. Therefore, coding approaches based on appearances and features are suggested for summarizing and generalizing experiences from the successfully performed operations. The multisensor trajectories and the motor signals are "grounded" in the learned operation sequences. Fig. 4 depicts the instruction for building an "elevator control" and the corresponding sensor trajectory. 

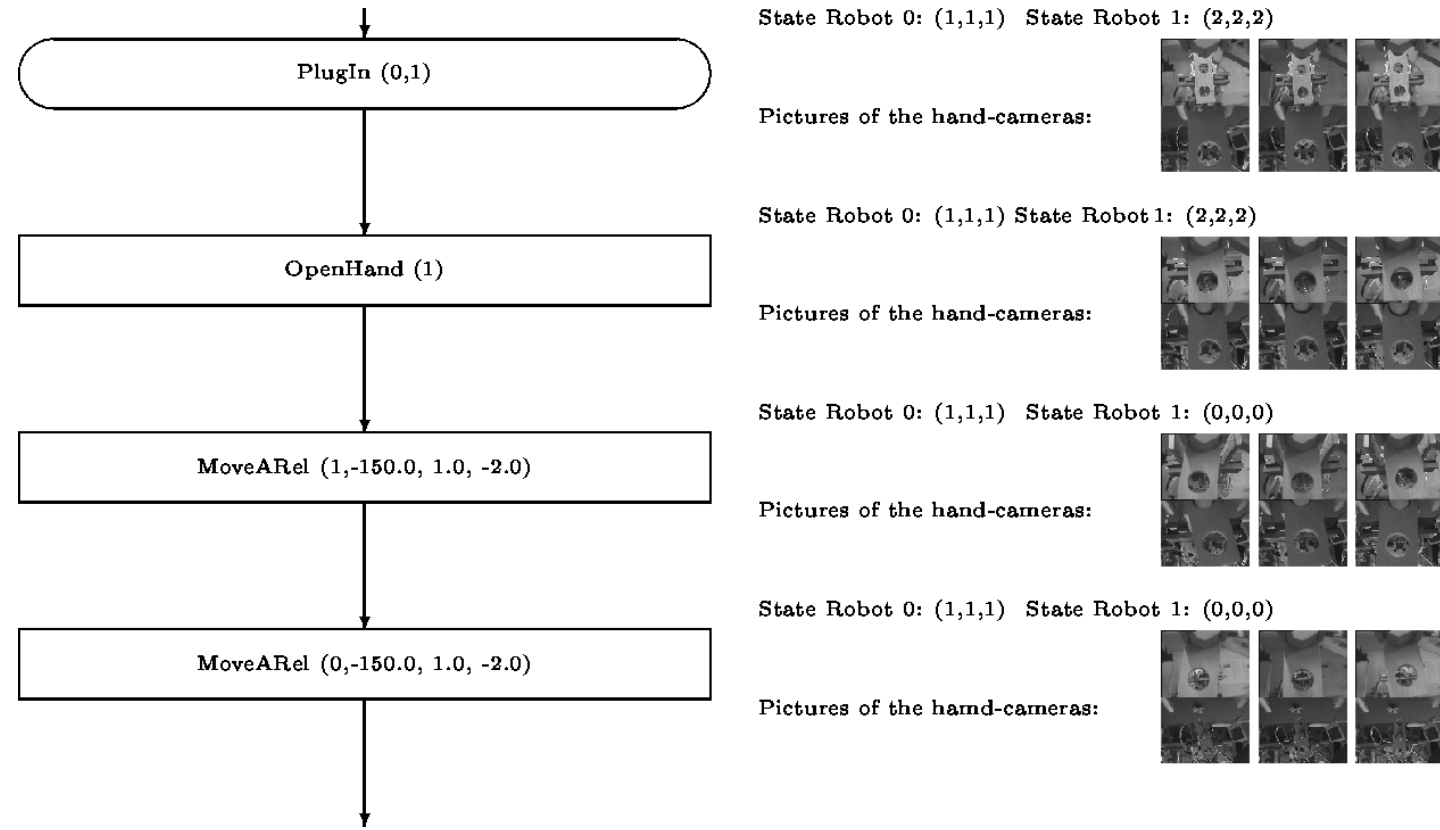

State Robot 0: $(1,1,1)$ State Robot 1: $(2,2,2)$

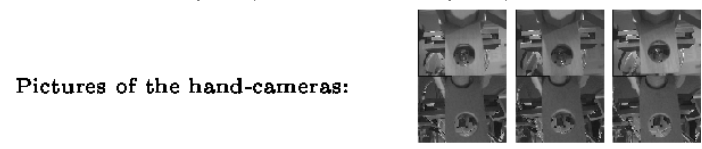

State Robot 0: $(1,1,1)$ State Robot 1: $(0,0,0)$

Pictures of the hand-cameras:

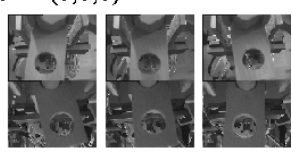

State Robot 0: $(1,1,1)$ State Robot 1: $(0,0,0)$

Pictures of the hand-cameras:

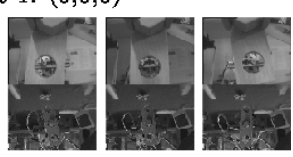

Fig. 4. Instruction sequence with sensor trajectory for building an "elevator control." The parameters of the instructions "PlugIn," "OpenHand," and "MoveARel" (relative movement along the approach axis of the tool frame) can be flexibly set. Different instruction sequences leading to the same aggregate are fused by a generic approach.

\section{Execution Functions}

Sequences are executed by the sequencer, which activates different skills on the execution level.

1) Robot Skill Library: The complexity of the skills can range from opening the hand to collision-free control of the two arms to a meeting point. Advanced skills are composed of one or more basic skills. Generally, three different kinds of skills are defined.

1) Motor skills: Open and close gripper; Drive joint to; Drive arm to; Rotate gripper; Move arm in approach direction; Move camera, etc.

2) Sensor skills: Get joint; Get position in world; Get force in approach direction; Get torques; Check if a specific position is reachable; Take a camera picture; Detect object; Detect moving robot; Track an object, etc.

3) Sensorimotor skills: Force-guarded motion; $\mathrm{Vi}$ sion-guided gross movement to a goal position; Visual servoing of the gripper to optimal grasping position, etc.

2) Control by a Neuro-Fuzzy Model: We developed a universal neuro-fuzzy method as the underlying model for robot skill learning [12]. Our experimental results show under the most diverse conditions that we can extract geometric features based on the calculations of moments to encode the positioning information and to find nongeometric parameters based on combining principal components. Therefore, if the input is high dimensional, an efficient dimension reduction can be achieved by projecting the original input space into a minimal subspace.

Variables in the subspace can be partitioned by covering them with linguistic terms (the right part of Fig. 5). In the following implementations fuzzy controllers constructed according to the B-spline model are used [11]. This model can be classified as an

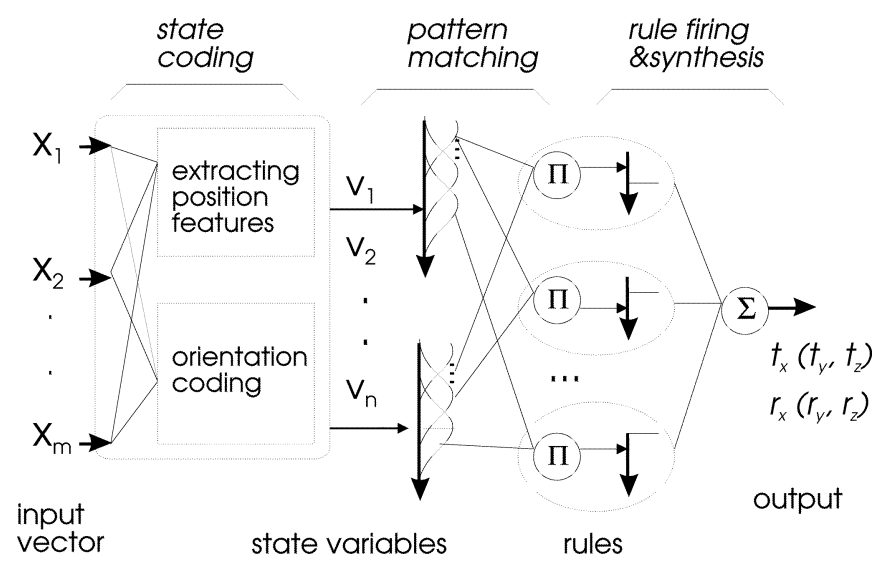

Fig. 5. Perception-action mapping is realized based on a neuro-fuzzy model.

adaptive, universal function approximator using regularization approaches. It provides an ideal implementation of the cerebellar model articulation controller (CMAC) model.

We define linguistic terms for input variables with B-spline basis functions and for output variables with singletons. This method requires fewer parameters than other set functions such as trapezoid, Gaussian function, etc. The output computation is very simple and the interpolation process is transparent. We also achieved good approximation capabilities and rapid convergence of the B-spline controllers. Both self-supervised and reinforcement learning have been applied to this model to realize most of the sensorimotor skills [12].

\section{Layered Learning}

Learning the interplay of perception, positioning, and manipulation is the foundation of a smooth execution of a command sequence of a human instructor. If a command refers to 


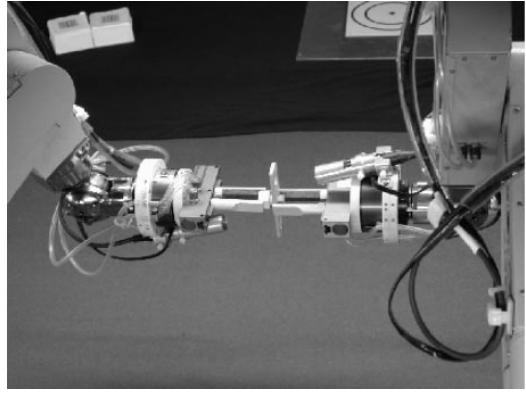

(a)

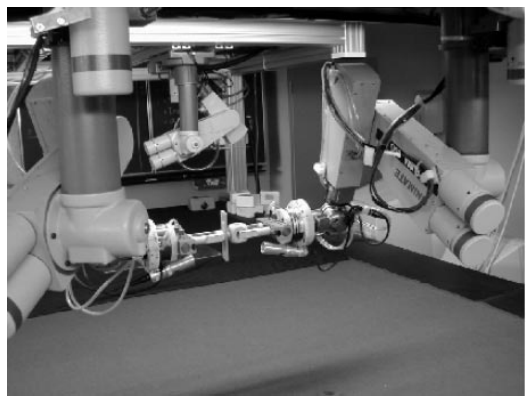

(c)

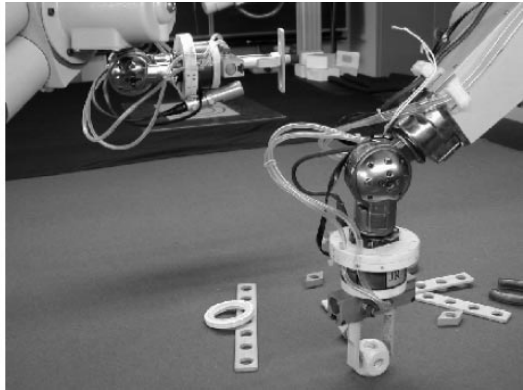

(b)

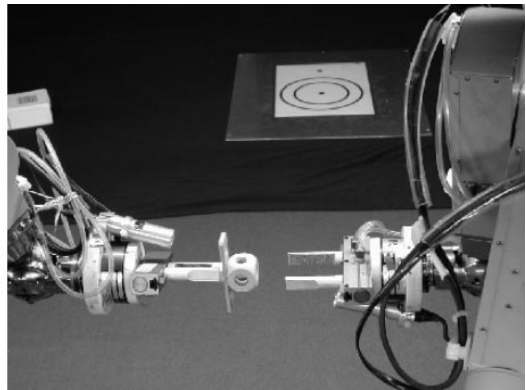

(d)

Fig. 6. Learned assembly process for building a simple aggregate. (a) Mounting a ledge. (b) Grasping a cube. (c) Mounting the cube. (d) Goal state reached.

an EO, the disambiguation of the instruction based on multimodality is the key process. The autonomous sensor-based execution of these instructions requires adaptive, multisensor-based skills with an understanding of a certain amount of linguistic labels. If complex instructions are used, however, the robot system should possess capabilities of skill fusion, sequence generation, and planning. It is expected to generate the same result after a repeated instruction even if the starting situation has changed. The layered learning approach is the scheme to meet this challenge.

Under this concept, tasks are decomposed from high to low level. Real situated sensor and actuator signals are located on the lowest level. Through task-oriented learning, the linguistic terms for describing the perceived situations as well as robot motions are generated. Skills for manipulation and assembly are acquired by learning on this level using the abovementioned neuro-fuzzy model. Furthermore, the learning results on the lower levels serve as the basis of the higher levels such as EO's, sequences, strategies, planning, and further cognitive capabilities.

To learn the operation sequences automatically for two arms, we developed a method for learning cooperative tasks. If a single robot is unable to grasp an object in a certain orientation, for example, it can only continue with the help of other robots. The grasping can be realized by a sequence of cooperative operations that re-orient the object. Several sequences are needed to handle the different situations in which an object is not graspable for the robot. It is shown that a distributed learning method based on a Markov decision process can learn the sequences for the involved robots, a master robot that needs to grasp and a helping robot that supports it with the re-orientation. A novel state-action graph scheme is used to store the reinforcement values of the learning process [4]. Fig. 6 shows an assembly process

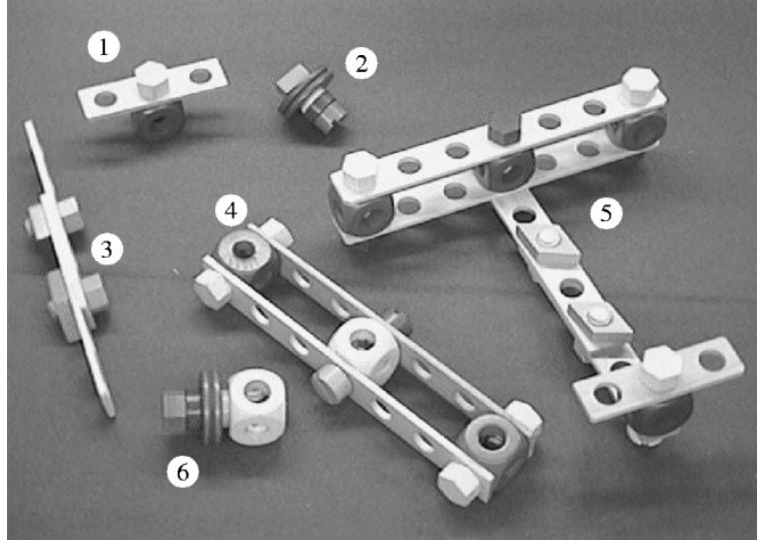

Fig. 7. Finished aggregates that can currently be built in multimodal dialogues by the SAC assembly robot.

learned by the state-action graph representation. The aggregate composed of a screw, a ledge and a cube is to be assembled. We use the object description and its graph matching algorithms to find out whether the object to construct is a subassembly of the goal aggregate (positive reward) or not (negative reward). This will give a reward whenever a part is successfully attached to the growing aggregate.

\section{E. Experimental Results}

Fig. 7 shows typical aggregates that can be built with the setup as developed up to now. Here, we briefly describe a sample dialogue which was carried out between the SAC and the HC in order to build the "elevator control" aggregate (number 1 in Fig. 7) of the aircraft out of three elementary objects. The objects were laid out on the table, and there were many more objects positioned in an arbitrary order on the table than necessary. 
The HC had a complete image in mind of what the assembly sequence should be. Alternatively, one could have used the assembly drawings in the construction kit's instructions and translated them into NL.

The first SAC input request is output after it checked that all modules of the setup are working properly. The necessary classification and subsequent steps are based on the color image obtained from the overhead color camera. After the SAC finds out if all objects are present and after going through an optional object naming procedure, the HC input "Take a screw!" first triggers the action planner, which decides which object to grasp and which robot to use. Since the HC did not specify either of these parameters, both are selected according to the principle of economy. In this case, they are chosen so as to minimize robot motion. The motion planner then computes a trajectory, which is passed to the RCCL/RCI subsystem (Robot Control C Library/Real-Time Control Interface). Since there are enough bolts available, the SAC issues its standard request for input once the bolt is picked up.

An HC input "Now, take the three-hole slat!" results in the other robot picking up the slat. Before this may happen, however, it has to be cleared up, which slat to take (SAC: "I see more than one such slat" and HC: "Take this one!" (points to one)). This involves the incorporation of the gesture recognizer. Under the instruction "Screw the bolt through the slat," the screwing is triggered, involving the peg-in-hole EO mentioned above followed by the screwing EO. For reasons of space, the subsequent steps of the dialogue have to be omitted here; they show how error handling and many other operations can be performed-most of which humans are not aware of when they expect machines to do "what I mean."

\section{FUTURE WORK}

Among the many topics to be explored in future research, some important ones can be listed as follows.

- Seamless communicator-Interfaces will be closely coupled with planning and monitoring. Ideal action needs to be inferred based on motion and action planning while considering the context and the human preference.

- Active intention detection based on multiple cues-Speech, gesture, and motion sequences (human demonstrations) will be integrated and combined with contexts, knowledge, and personal preference. The cross-modal interplay will be investigated. Since the system resources are limited, sensory input needs to be selected by using factor analysis, signal synthesis, and tracking focus of interests.

- General human perception-Human motions are captured without using artificial markers. Wide-range, active camera configurations are applied to human recognition and precise gaze perception, even under conditions like low-quality input and occlusions. The robustness of the voice input in real environments should be significantly improved. This task is even more challenging if non-close-speaking microphones are used.
- Grounded learning of multisensor events, sequences, and human activities-Long-term memory should be learned from short-term memory so that symbols, sequences, names, and attributes are anchored in the real sensor/actuator world. To enable the arbitrary transition between digital measurements and concepts, symbolic sparse coding, granular computing, fuzzy sets, and rough sets will be investigated and integrated. The sensor capability can be extended by using linguistic modeling of human perception and sensor fusion so that information which is difficult to measure, incomplete, or noisy can be perceived. Learning on the higher level should be conducted to select action strategies and to generate intelligent dialogues. This will require the tight integration of more components and more knowledge. The combination of grounded learning and communication will make human-robot interaction work like interaction with a growing child, which will be really entertaining.

\section{REFERENCES}

[1] R. Bischoff and V. Graefe, "Integrating vision, touch and natural language in the control of a situation-oriented behavior-based humanoid robot," in Proc. IEEE Int. Conf. Systems, Man, and Cybernetics, vol. 2, Tokyo, Japan, 1999, pp. 999-1004.

[2] R. A. Brooks, C. Breazeal, M. Marjanovic, and B. Scassellati, "The Cog project: building a humanoid robot," in Computation for Metaphores, Analogy and Agents, C. L. Nehaniv, Ed. Berlin, Germany: Springer-Verlag, 1999, vol. 1562, Lecture Notes in Computer Science, pp. 52-87.

[3] A. Clark and R. Grush, "Toward a cognitive robotics," Adapt. Behav., vol. 7, no. 1, pp. 5-16, 1999.

[4] M. Ferch and J. Zhang, "Learning cooperative grasping with the graph representation of a state-action space," J. Robot. Auton. Syst., vol. 38, no. 3-4, pp. 183-196, 2002.

[5] C. Grangle and P. Suppes, Language and Learning for Robots. Stanford, CA: CSLI, 1994.

[6] T.Th. Laengle, T. C. Lueth, E. Stopp, and G. Herzog, "Natural language access to intelligent assembly robots: explaining automatic error recovery," in Artificial Intelligence: Methodology, Systems, Applications, A. M. Ramsay, Ed. Amsterdam, The Netherlands: IOS, 1996, pp. 259-267.

[7] G. Lakoff, Women, Fire, and Dangerous hings: What Categories Reveal About the Mind. Chicago, IL: Univ. of Chicago Press, 1990.

[8] R. Moratz, H. Eikmeyer, B. Hildebrandt, A. Knoll, F. Kummert, G. Rickheit, and G. Sagerer, "Selective visual perception driven by cues from speech processing," in Proc. EPIA'95, 1995, pp. 63-72.

[9] K. R. Thorissen, "Communicative humanoids-A computational model of psychosocial dialogue skills," Ph.D. dissertation, Media Lab., Massachusetts Inst. Technol., Cambridge, MA, 1997.

[10] J. Weng, C. H. Evans, W. S. Hwang, and Y.-B. Lee, "Developmental robots: Theory, method, and experimental results," in Proc. Symp. Humanoid Robots, Tokyo, Japan, Oct. 8-9, 1999, pp. 57-64.

[11] J. Zhang and A. Knoll, "Constructing fuzzy contro1Iers with B-spline models-principles and applications," Int. J. Intell. Syst., vol. 13, no. 2/3, pp. 257-285, Feb./Mar. 1998.

[12] — "A neuro-fuzzy learning approach to visually, guided 3D positioning and pose control of robot arms," in Biologically Inspired Robot Behavior Engineering, R. Duro, J. Santos, and M. Grana, Eds. Berlin, Germany: Springer-Verlag, 2001.

[13] J. Zhang, Y. von Collani, and A. Knoll, "Interactive assembly by a two-arm robot agents," J. Robot. Auton. Syst., vol. 29, pp. 91-100, 1999. 


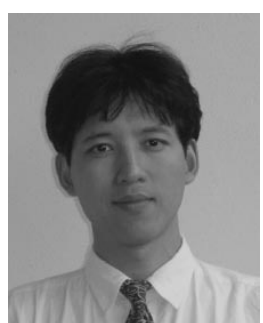

Jianwei Zhang (M'92) received the Bachelor of Engineering degree with distinction and the Master of Engineering degree from the Department of Computer Science, Tsinghua University, Beijing, China, in 1986 and 1989, respectively, and the Ph.D. degree from the Institute of Real-Time Computer Systems and Robotics, Department of Computer Science, University of Karlsruhe, Karlsruhe, Germany, in 1994.

From August 1994 to July 2002, he was an Assistant Professor in the Department of Technology, University of Bielefeld. Since August 2002, he has been a Full Professor and the Director of the Technical Aspects of Multimodal Systems Group, Department of Computer Science, University of Hamburg, Hamburg, Germany. His research interests are robot control architecture, planning, sensor-based manipulation, robot learning, man-machine interfaces, and applications of neuro-fuzzy systems. In these areas, he has authored more than 90 journal and conference papers, technical reports, five book chapters, and two research monographs. He leads several projects in the collaborative research center "Situated Artificial Communicators" and coordinates the European Interest Group on "Skill Learning/Multimodal Interaction." He also leads several application projects in laboratory service robots, mobile robots, and sensing devices.

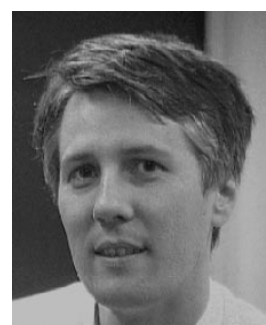

Alois C. Knoll received the Diploma (M.Sc.) degree in electrical engineering from the University of Stuttgart, Stuttgart, Germany, in 1985, and the Ph.D. degree (summa cum laude) in computer science from the Technical University of Berlin (TU Berlin), Berlin, Germany, in 1988.

He served on the faculty of the Computer Science Department of TU Berlin until 1993, when he qualified for teaching computer science at the university level (habilitation). He then joined the Technical Faculty of the University of Bielefeld, where he was a Full Professor and the Director of the research group Technical Informatics until 2001. In March 2001, he was appointed to the Board of Directors of the Fraunhofer-Institute for Autonomous Intelligent Systems. Since autumn 2001, he has been a Professor in the Computer Science Department, Technical University of Munich, Munich, Germany. His research interests include sensorbased robotics, multi-agent systems, data fusion, adaptive systems, and multimedia information retrieval. In these fields, he has authored more than 80 published technical papers, and has guest-edited international journals. He has been part of (and has coordinated) several large-scale national collaborative research projects (funded by the EU, the DFG, the DAAD, and the state of North-Rhine-Westphalia).

Prof. Knoll initiated and was the Program Chairman of the First IEEE Robotics and Automation Society Conference on Humanoid Robots (IEEE-RAS/RSJ Humanoids 2000), held at the Massachusetts Institute of Technology, Cambridge, in September 2000. He is a Member of the Gesellschaft fuer Informatik. 